\title{
Fractional flow reserve, quantitative flow ratio, and instantaneous wave-free ratio: a comparison of the procedure-related dose of ionising radiation
}

\author{
Greta Ziubryte, Gediminas Jarusevicius \\ Department of Cardiology, Hospital of Lithuanian University of Health Sciences Kaunas Clinics, Institute of Cardiology, \\ Lithuanian University of Health Sciences, Kaunas, Lithuania
}

Adv Interv Cardiol 2021; 17, 1 (63): 33-38

DOI: https://doi.org/10.5114/aic.2021.104765

\begin{abstract}
A bstract
Introduction: The development of interventional cardiology increases the number of invasive procedures which are inevitably associated with increased exposure to ionizing radiation and associated risks. A percutaneous coronary intervention ( $\mathrm{PCI})$ substantiated by evaluation of the coronary artery lesion's functional significance is recommended by both European and American cardiologists. Nevertheless, the prevalence of physiology-guided PCls does not exceed $10 \%$ all over the globe.

Aim: To identify the physiology evaluation method which is associated with the lowest exposure to ionising radiation.

Material and methods: Anonymised data of 421 patients with stable angina pectoris for whom elective coronary artery angiography followed by physiological assessment of intermediate coronary artery stenosis was performed were prospectively included in this study. Only diagnostic-procedure-related data of dose of ionizing radiation were analysed. Physiological assessment of coronary artery lesions was performed by fractional flow reserve (FFR), quantitative flow ratio (QFR), or instantaneous wave-free ratio (iFR).

Results: Compared to FFR as a reference, fluoroscopy time (FT) was almost half in QFR and almost double in iFR, $p<0.001$. QFR was associated with more than 3 times shorter FT compared to iFR. The dose area product was $663.87 \pm 260.51 \mathrm{cGy} / \mathrm{cm}^{2}(p=0.03)$ lower in QFR compared to iFR.

Conclusions: QFR is associated with significantly reduced exposure to ionising radiation compared to both FFR and iFR. Therefore, wider QFR application in clinical practice could eliminate any additional exposure to ionising radiation and increase the prevalence of physiology-guided coronary artery revascularization.
\end{abstract}

Key words: fractional flow reserve, ionising radiation, quantitative flow ratio, instantaneous wave-free ratio, physiology-guided coronary revascularisation.

Su m m a ry

Coronary interventions substantiated by the physiological evaluation of coronary lesions are associated with better outcomes; therefore, they are supported by experts worldwide. Nevertheless, the use of physiological assessment methods remains insufficient, mainly due to procedure prolongation, increased dose of ionising radiation, and possible complications. This study was designed to identify the method associated with the lowest exposure to ionising radiation. A novel, minimally invasive fractional flow reserve value computation from an ordinary angiogram method - quantitative flow ratio (QFR) - has shown a significant reduction in expose to ionising radiation compared to pressure-wire-based methods. Hence, wider QFR application in clinical practice may safely increase the prevalence of physiology-guided revascularisation.

\section{Introduction}

The development of interventional cardiology increases the prevalence of invasive procedures which are inevitably associated with increased exposure to ionising radiation and associated risks for the physicians [1].
Despite adequate radiation protection, interventional cardiologists among all physicians working in radiology facilities receive the highest amount of ionising radiation $[2,3]$. Seymour et al. estimated that during his or her career, an interventional cardiologist is exposed to a cu-

\section{Corresponding author:}

Greta Ziubryte, Department of Cardiology, Hospital of Lithuanian University of Health Sciences Kaunas Clinics, Institute of Cardiology, Lithuanian University of Health Sciences, Kaunas, Lithuania, phone: +37 061110128, e-mail: greta.ziubryte@gmail.com

Received: 6.07.2020, accepted: 30.10.2020. 
mulative dose of ionising radiation equal to 10000 chest $X$-rays $[1,4]$. In contrast, for the most sensitive organ, the brain, the effect of the received dose of ionising radiation increases 5-fold [4], which could lead to increased risk of development of left side brain and neck tumours $[5,6]$.

A percutaneous coronary intervention ( $\mathrm{PCl}$ ) substantiated by the evaluation of coronary artery (CA) lesion's functional significance is strongly recommended by both the European Society of Cardiology/European Association for Cardio-Thoracic Surgery (ESC/EACTS) and the American College of Cardiology (ACC) [7-9]. According to the latest guidelines on myocardial revascularization, the method of choice for intermediate coronary artery lesions' functional evaluation is fractional flow reserve (FFR) followed by an instantaneous wave-free ratio (iFR) [7-9]. They are pressure-wire-based methods extending the costs and the duration of the procedure [9]. Also, the necessity of a stiff wire insertion through a tight lesion might be complicated by a failure to pass through the lesion or in forceful manoeuvres by a plaque rupture, a CA dissection or perforation [9]. Moreover, an FFR measurement requires intravenous or intracoronary adenosine injection which occasionally may cause drug-related adverse reactions including dyspnoea, chest discomfort, bradycardia, atrioventricular blocks, ventricular arrhythmias, cardiac arrest, asystole and even death [10]. An iFR is advantageous due to redundancy of adenosine due to exploitation of the natural heart cycle hyperaemic phase [11]. Its performance and agreement with FFR has been proved in numerous clinical trials $[12,13]$. Nevertheless, the application of pressure-wire-based methods remains extremely low and, in the best scenario, does not exceed $10 \%$ of all PCls all over the world [9, 14-16]. Seeking a functional evaluation method that is as non-invasive as possible, a novel minimally invasive quantitative flow ratio (QFR) method based on three-dimensional (3D) coronary artery reconstruction from an ordinary diagnostic coronary artery angiography (CAG) images and FFR value computation by applying specific mathematical equations was developed a couple years ago [9, 15-17] and has demonstrated promising results. Previous studies have proved excellent agreement separately between FFR and both iFR and QFR [9, 12, 13, 15-26]. However,

Table I. Inclusion criteria

\begin{tabular}{l} 
Performed CAG \\
\hline Visually estimated lumen stenosis of $35-90 \%$ \\
\hline Investigated CA diameter above $2 \mathrm{~mm}$ in size \\
\hline Indications for treatment of the investigated CA \\
\hline Physiology assessment performed by either FFR, QFR or iFR \\
Available procedure data on fluoroscopy time and dose area prod- \\
uct \\
CA - coronary artery, CAG - coronary artery angiography, FFR - fractional flow \\
reserve, iFR - instantaneous wave-free ratio, QFR - quantitative flow ratio.
\end{tabular}

the data comparing all three methods at any aspect are scarce. Hence, we designed this study to compare the dose of ionising radiation received during diagnostic CAG accompanied by performance of FFR, QFR or iFR for CA lesions' physiological evaluation.

\section{Aim}

To compare the dose of ionising radiation received during CAG accompanied by performance of FFR, QFR or iFR for CA lesions' physiological evaluation.

\section{Material and methods}

\section{Study population}

After study approval by the Regional Ethical Committee, anonymized data of 421 patients who were admitted to our hospital due to stable angina pectoris between $1^{\text {st }}$ January 2018 and $1^{\text {st }}$ March 2020 were prospectively included in our single-centre observational study. For all included patients, an elective CAG followed by functional assessment of intermediate (lumen diameter stenosis of 35-90\%) CA stenosis was performed. For the physiological evaluation, one of three methods (FFR, QFR, or iFR) was chosen according to the operator's preference.

All included patients met all the inclusion criteria given in Table I. Patients who did not meet at least one inclusion criterion were excluded from the analysis.

\section{Coronary angiography technique}

CAG was performed in standard fashion, obtaining images after intracoronary nitrate administration. The right radial approach was chosen for all procedures. All CAGs were performed using GE Healthcare Innova Cardiovascular Imaging System machines. Standard coronary artery angiograms in our catheterization laboratory employ certain angulations including anteroposterior (AP) caudal (about $30^{\circ}$ ) and AP cranial (about $30^{\circ}$ ), left anterior oblique (LAO) cranial (both between $20^{\circ}$ and $30^{\circ}$ ), right anterior oblique (RAO) cranial (both between $20^{\circ}$ and $30^{\circ}$ ) and RAO caudal (both between $20^{\circ}$ and $30^{\circ}$ ) for the left coronary artery and LAO cranial (both between $15-30^{\circ}$ ) and AP cranial (about $20^{\circ}$ ) or RAO cranial (both between $\left.10-30^{\circ}\right)$ for the right coronary artery. For QFR analyses, dedicated angulations [27] were acquired if necessary. The chosen frame rate of 7.5 frames per second is used for fluoroscopy and 15 frames per second for cinematography modes. A standard diagnostic CAG procedure was followed by a physiological evaluation procedure.

\section{Physiology evaluation techniques}

After engagement of the $6 \mathrm{~F}$ guide catheter, the pressure wire was placed at the aortic ostium of either the right or left coronary artery under fluoroscopy mode guidance, and then the calibration and equalization to aortic pressure were performed. Later, the pressure wire 
was placed distally to the index lesion. The position of the wire was recorded in cinematography mode. In total, $0.28 \mathrm{mg}$ of intracoronary adenosine were injected for maximal hyperaemia induction during every FFR measurement. After 5-10 s, the FFR was recorded. Then the guiding catheter was flushed with saline, and the FFR measurements repeated twice more. When performing iFR measurements, intravenous adenosine injection was omitted. A pullback record was performed in both FFR and iFR measurements. Online QFR analyses were performed using the certified software package QAngio-XA 3D, version 2.0 (Medis Medical Imaging Systems, Leiden, The Netherlands) from coronary artery angiograms, acquired following previously described recommendations [24]. All functional assessments were performed by an experienced interventional cardiologist and repeated at least three times to avoid any possible mismatches; averaged values were included in the analysis.

\section{Measures of ionising radiation dose}

The ionising radiation dose was evaluated by dose area product (DAP) and total fluoroscopy time (FT). Data were collected from index diagnostic procedure protocols obtained from the local database.

Only diagnostic-procedure-related data were analysed; hence the dose of ionising radiation exposed during $\mathrm{PCl}$ s was not included in the analysis.

\section{Statistical analysis}

Categorical data were presented as numbers and percentages. Continuous variables were normally distributed, presented as mean with standard deviation ( \pm ) and were compared using the unpaired Student's two-sided $t$-test. One-way ANOVA was used to assess potential differences between groups. Statistical analysis was performed using the software package SPSS version 20.0 (IBM Corp., Armonk, NY, USA). The chosen level of significance was $p<0.05$.

\section{Results}

Of all 421 investigated procedures 192 (45.6\%) were FFRs, 134 (31.8\%) were QFRs and 95 (22.6\%) were iFRs (Figure 1).

The mean FT was $5.97 \pm 0.32 \mathrm{~min}$ in the FFR group, $2.81 \pm 0.23 \mathrm{~min}$ in the QFR group, and $9.30 \pm 0.62 \mathrm{~min}$ in the iFR group (Figure 2). Compared to FFR as a reference, FT was almost half in QFR and almost double in iFR, $p<$ 0.001 . Moreover, it was found that the duration of QFR analyses is more than 3 times shorter compared to iFR measurements, $p<0.001$.

The mean DAP was $2040.57 \pm 151.64 \mathrm{cGy} / \mathrm{cm}^{2}$ in the FFR group, $1587.85 \pm 136.76 \mathrm{cGy} / \mathrm{cm}^{2}$ in the QFR group and $2251.72 \pm 211.77 \mathrm{cGy} / \mathrm{cm}^{2}$ in the iFR group (Figure 3). DAP was lower in QFR compared to iFR with the mean difference of $663.87 \pm 260.51 \mathrm{cGy} / \mathrm{cm}^{2}, p=0.03$. No sig-

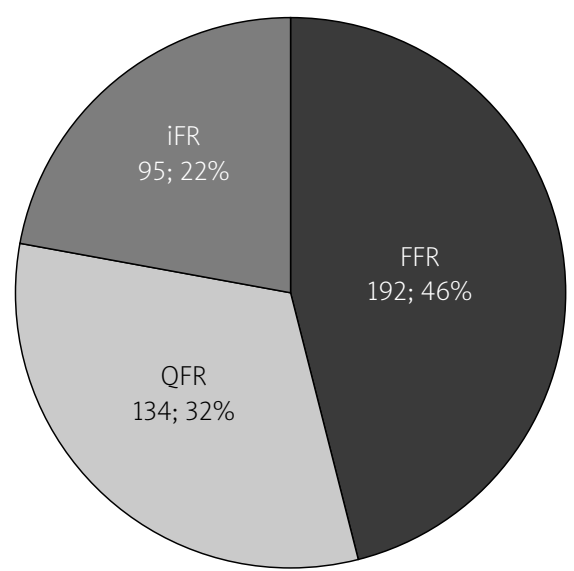

Figure 1. Distribution of physiology evaluation methods

nificant differences between other groups were found. The study design and results are summarized in Figure 4.

\section{Discussion}

Development of interventional cardiology with an increasing number of procedures over the past decade

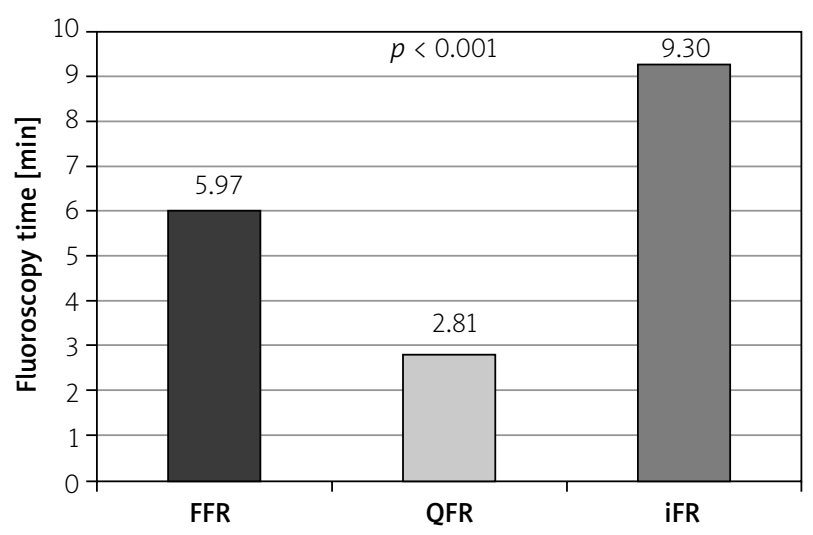

Figure 2. Comparison of fluoroscopy time in FFR, quantitative flow ratio and instantaneous wavefree ratio groups

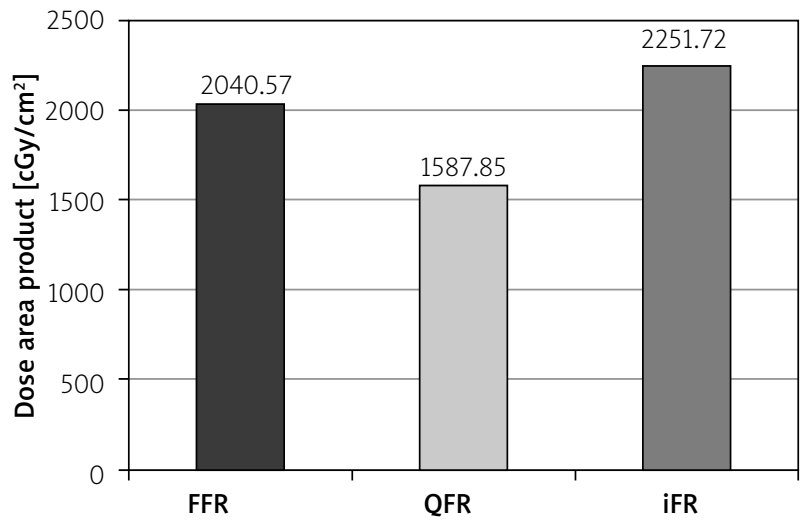

Figure 3. Comparison of dose area product in FFR, quantitative flow ratio and instantaneous wavefree ratio groups 


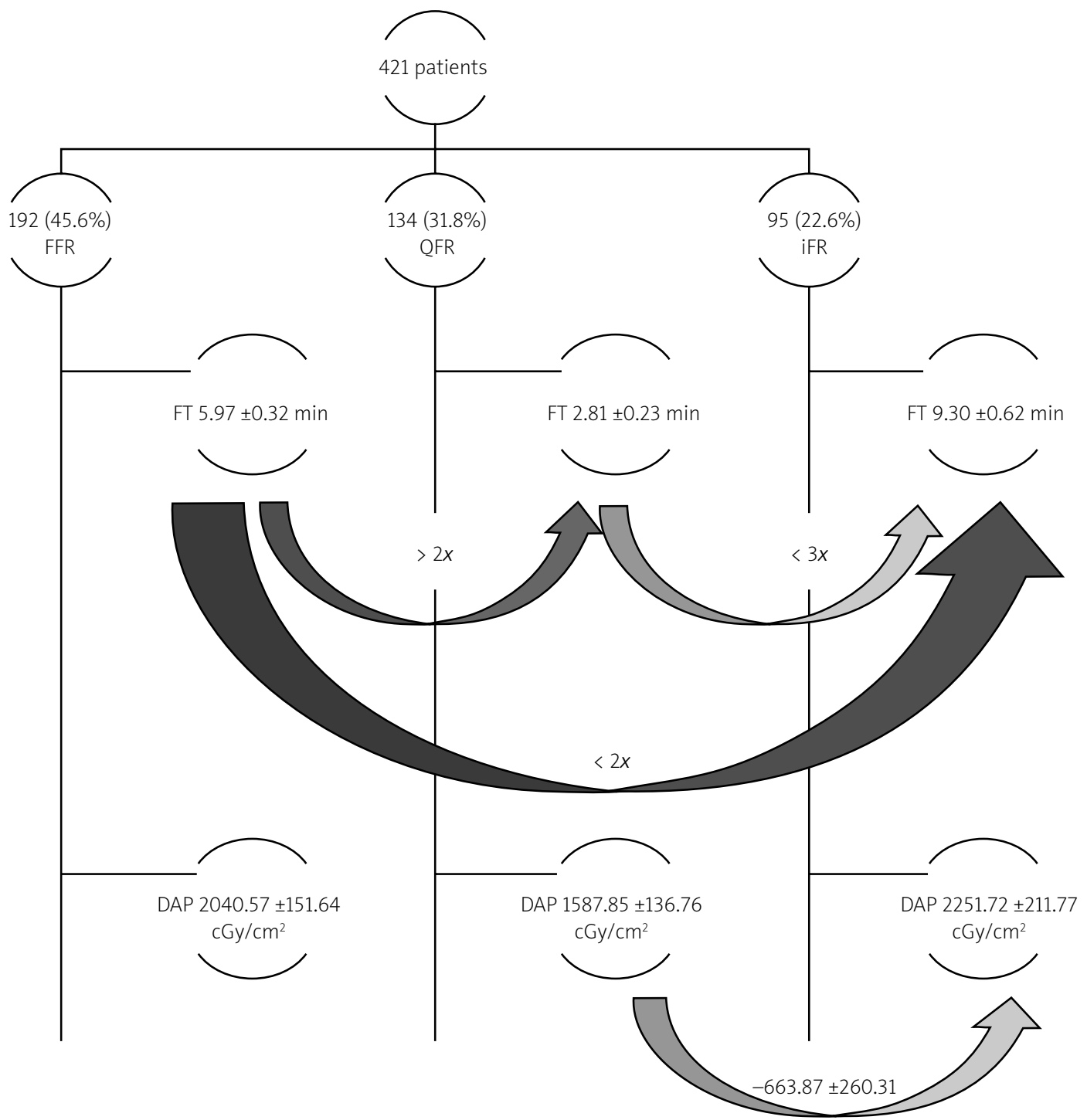

Figure 4. Schematic study methods and results. Curved arrows mark the direction of comparison between two physiological assessment methods, number above the arrows shows either fold difference or numerical expression of the difference between compared values

$D A P$ - dose area product, FFR - fractional flow reserve, FT - fluoroscopy time, iFR - instantaneous wave-free ratio, QFR - quantitative flow ratio.

[27-30] is inevitably associated with increased requirement for physiology guidance of $\mathrm{PCl}[7,8]$. The use of any of pressure-wire-based physiology evaluation method (FFR or iFR) remains limited in clinical practice, likely due to the requirement of stiff pressure-wire intracoronary instrumentations and its association with complications in addition to prolongation of the procedure time and increase of the economic aspects [9, 31-34]. The newly developed wireless FFR value computation method QFR seems to be beneficial due to the lack of need of adenosine injections and intracoronary instrumentation of pressure-wire resulting in a shorter procedure and fluoroscopy time [9], which was double in the FFR group and three times longer in the iFR group compared to QFR analysis in this study. Moreover, FT compared to FFR was almost double in iFR, most likely due to intracoronary in- strumentation with a stiffer, less torque and less flexible pressure-wire dedicated for iFR. It normally takes longer to pass through tight lesions with stiffer wire. This QFR benefit might be explained by the lack of need of any intracoronary manoeuvres under fluoroscopy guidance $[15,24,35]$. That certainly results in shorter FT and lower DAP. It is known that DAP is closely related to angiography technique and patients' body constitution. Unfortunately, patients' physical characteristics were not included in this analysis. Accordingly, associations between the body constitution and exposure to ionising radiation were not investigated. Therefore, DAP was considered as a less reliable parameter for evaluation of the ionising radiation dose associated with the used physiological evaluation method in this study. Nevertheless, DAP was lower in QFR compared to iFR. A higher dose of ionising 
radiation is necessary to obtain appropriate quality angiograms for patients with increased body mass index (BMI), especially when $\mathrm{BMI}$ is over $30 \mathrm{~kg} / \mathrm{m}^{2}[3,36,37]$. Cinematography mode requires the double frame rate compared to fluoroscopy mode; therefore cinematography replacement by fluoroscopy is supported by several scientists [38]. Even though significantly longer FT in FFR and iFR groups, possibly due to intracoronary instrumentations of pressure-wire, it can be assumed that proper angiograms may reduce FT and DAP in addition to a shorter overall procedure time when QFR is chosen for physiology evaluation [15, 18-26]. The use of QFR could reduce to the minimum the amount of additional ionising radiation during physiological assessment procedures without loss of diagnostic accuracy [15, 18-26]. Eventually, the application of QFR significantly reduces total FT compared to pressure-wire-based methods and substantially reduces the total procedure time [35]. Wider application in clinical practice of QFR as a preferred physiology-evaluation method, in addition to the increase of physiologically guided $\mathrm{PCl}$, could eliminate any additional exposure to ionising radiation warranting the highest level of radiation protection for interventional cardiologists.

Despite its novelty comparing the exposure to ionising radiation during coronary artery physiological assessment procedures, the study has certain limitations. Data on patients' body constitutions and the number of diagnosed vessels were not included. Hence, we were not able to validate the impact of BMI on ionizing radiation increase during interventional procedures. Furthermore, the desire to reduce the exposure to ionising radiation during physiology assessment procedures raised the hypothesis of a possibility to perform qualified QFR analysis using images acquired under fluoroscopy mode. This is the subject for further studies. The study is being continued, including omitted parameters.

\section{Conclusions}

Physiological assessment by QFR, in addition to its reproducibility, convenience, and accuracy, is associated with significantly reduced exposure to ionizing radiation compared to both FFR and iFR. Therefore, wider QFR application in clinical practice and consideration of it as the first-choice method for physiological evaluation, in addition to increase of physiology-guided coronary artery revascularization, could eliminate any additional exposure to ionizing radiation. Moreover, acceptance of standard QFR projections in routine clinical practice may enable wider use of the method and may significantly enhance QFR benefits.

\section{Conflict of interest}

The authors declare no conflict of interest.

\section{References}

1. Health impacts of Radiation Exposure During PCl. Seymour M. Available at: https://www.cathlabdigest.com/article/Health-Impacts-Radiation-Exposure-During-PCI Accessed at April 15, 2020.

2. Goyes JCR, Gomez NJ, Restrepo VJ, et al. Radiation exposure in a group of interventional cardiologists in a high volume $\mathrm{PCl}$ center. Interv Cardiol 2018; 10: 73-9.

3. Kharji SA, Connell T, Bernier M, Eisenberg MJ. Ionizing radiation in interventional cardiology and electrophysiology. Can J Cardiol 2019; 35: 535-8.

4. Picano E, Vano E, Domenici L, et al. Cancer and non-cancer brain and eye effects of chronic low-dose ionizing radiation exposure. BMC Cancer 2012; 12: 157.

5. Roguin A, Goldstein J, Bar O, Goldstein JA. Brain and neck tumors among physicians performing interventional procedures. Am J Cardiol 2013; 111: 1368-72.

6. Roguin A, Goldstein J, Bar O. Brain tumours among interventional cardiologists: a cause for alarm? Report of four new cases from two cities and a review of the literature. Eurolntervention 2012; 7: 1081-6.

7. Neumann FJ, Sousa-Uva M, Ahlsson A, et al. ESC Scientific Document Group. 2018 ESC/EACTS Guidelines on myocardial revascularization. Eur Heart J 2019; 40: 87-165.

8. Patel MR, Calhoon JH, Dehmer GJ, et al. ACC/AATS/AHA/ASE/ ASNC/SCAI/SCCT/STS 2017 Appropriate Use Criteria for Coronary Revascularization in Patients With Stable Ischemic Heart Disease: A Report of the American College of Cardiology Appropriate Use Criteria Task Force, American Association for Thoracic Surgery, American Heart Association, American Society of Echocardiography, American Society of Nuclear Cardiology, Society for Cardiovascular Angiography and Interventions, Society of Cardiovascular Computed Tomography, and Society of Thoracic Surgeons. J Am Coll Cardiol 2017; 69: 2212-41.

9. Westra J, Tu S, Campo G, et al. Diagnostic performance of quantitative flor ratio in prospective enrolled patients: an individual patient-data meta-analysis. Catheter Cardiovasc Interv 2019; 94: 693-701.

10. Zaleska M, Koltowski L, Maksym J, et al. Quantitative flow ratio and fractional flow reserve mismatch - clinical and biochemical predictors of measurements discrepancy. Adv Interv Cardiol 2019; 15: 301-7.

11. Baumann S, Chandra L, Skarga E, et al. Instantaneous wave-free ratio (iFR) to determine hemodynamically significant coronary stenosis: a comprehensive review. World J Caridiol 2018; 10 : 267-77.

12. Götberg M, Christiansen EH, Gudmundsdottir IJ, et al. Use of the instantaneous wave-free ratio or fractional flow reserve in $\mathrm{PCl}$. Am. Heart J 2015; 170: 945-50.

13. Andell P, Berntorp K, Christiansen EH, et al. Reclasification of treatment stratedy with instantaneous wave-free ratio and fractional flow reserve. A substudy from the iFR-SWEDENHEART trial. J Am Coll Cardiol Intv 2018; 11: 2084-94.

14. Davies JE, Sen S, Dehbi HM, et al. Use of the instantaneous wavefree ratio or fractional flow reserve in $\mathrm{PCI}$. N Engl J Med 2017; 376: 1824-34.

15. Westra J, Andersen BK, Campo G, et al. Diagnostic performance of in-procedure angiography-derived quantitative flow reserve compared to pressure-derived fractional flow reserve: the FAVOR II Europe-Japan study. J Am Heart Assoc 2018; 7: e009603. 
16. Westra J, Tu S, Winther S, et al. Evaluation of coronary artery stenosis by quantitative flow ratio during invasive coronary angiography: the WIFI II study (wire-free functional imaging II). Circ Cardiovasc Imaging 2018; 11: e007107.

17. Xu B, Tu S, Qiao S, et al. Diagnostic accuracy of angiographybased quantitative flow ratio measurements for online assessment of coronary stenosis. J Am Coll Cardiol 2017; 70: 3077-87.

18. Erbay A, Steiner J, Lauten A, et al. Assessment of intermediate coronary lesions by fractional flow reserve and quantitative flow ratio in patients with small-vessel disease. Catheter Cardiovasc Interv 2020; 96: 743-51.

19. Xing Z, Pei J, Huang J, et al. Diagnostic performance of QFR for the evaluation of intermediate coronary artery stenosis confirmed by fractional flow reserve. Brazilian J Cardiovasc Surg 2019; 34: 165-72.

20. Emori H, Kubo T, Kameyama T, et al. Diagnostic accuracy of quantitative flow ratio for assessing myocardial ischemia in prior myocardial infarction. Circulation 2018; 82: 807-14.

21. Yazaki K, Otsuka M, Kataoka S, et al. Applicability of 3-dimensional quantitative coronary angiography-derived computed fractional flow reserve for intermediate coronary stenosis. Circulation 2017; 81: 988-92.

22. Westra J, Tu S, Campo G, et al. Diagnostic performance of quantitative flow ratio in prospectively enrolled patients: an individual patient-data meta-analysis. Catheter Cardiovasc Interv 2019; 94: 693-701.

23. Ties D, van Dijk R, Pundziute G, et al. Computational quantitative flow ratio to assess functional severity of coronary artery stenosis. Int J Cardiol 2018; 271: 36-41.

24. Tu S, Westra J, Yang J, et al. Diagnostic accuracy of fast computational approaches to derive fractional flow reserve from diagnostic coronary angiography: the International Multicenter FAVOR Pilot Study. J Am Coll Cardiol Intv 2016; 9: 2024-35.

25. Xu B, Tu S, Qiao S, et al. Diagnostic accuracy of angiography-based quantitative flow ratio measurements for online assessment of coronary stenosis. J Am Coll Cardiol 2017; 70: 3077-87.

26. Chang Y, Chen L, Westra J, et al. Reproducibility of quantitative flow ratio: an inter-core laboratory variability study. Cardiol J 2020; 27: 230-7.

27. Peruzzo CA, Aimonetto S, Pisano F, et al. The contribution of interventional cardiology procedures to the population radiation dose in a 'health-care level I' representative region. Radiat Prot Dosimetry 2016; 168: 261-70.

28. Faxon DP, Williams DO. The changing face of interventional cardiology. Circ Cardiovasc Interv 2012; 5: 325-7.

29. Trianni A, Chizzola G, Toh H, et al. Patient skin dosimetry in haemodynamic and electrophysiology interventional cardiology. Radiat Prot Dosimetry 2005; 117: 241-6.

30. Picaso E, Santoro G, Vano E. Sustainability in the cardiac cath lab. Int J Cardiovasc Imaging 2007; 23: 143-7.

31. Johnson NP, Koo BK. Coronary psychology: do you believe? J Am Coll Cardiol Intv 2018; 11: 1492-4.

32. Hwang D, Choi KH, Lee JM, et al. Diagnostic agreement of quantitative flow ratio with fractional flow reserve and instantaneous wave-free ratio. J Am Heart Assoc 2019; 8: e011605.

33. Dehmer GJ, Weaver D, Roe MT, et al. A contemporary view of diagnostic cardiac catheterization and percutaneous coronary intervention in the United States: a report from the CathPCI Registry of the National Cardiovascular Data Registry, 2010 through June 2011. J Am Coll Cardiol 2012; 60: 2017-31.
34. Tebaldi M, Biscaglia S, Fineschi $M$, et al. Evolving routine standards in invasive hemodynamic assessment of coronary stenosis: the nationwide Italian SICI-GISE Cross-Sectional ERIS Study. J Am Coll Cardiol Intv 2018; 11: 1482-91.

35. Westra J, Andersen BK, Campo G, et al. Diagnostic performance of in procedure angiography-derived quantitative flow reserve compared to pressure-derived fractional flow reserve: the FAVOR II Europe-Japan study. J Am Heart Assoc 2018; 7: e009603.

36. Hwang J, Lee SY, Chon ML, et al. Radiation exposure in coronary angiography: a comparison of cineangiography and fluorography. Korean Circ J 2015; 45: 451-6.

37. Delewi R, Hoebers LP, Ramundadal T, et al. Clinical and procedural characteristics associated with higher radiation exposure during percutaneous coronary interventions and coronary angiography. Circ Cardiovasc Interv 2013; 6: 501-6.

38. Abbott JD. Controlling radiation exposure in interventional cardiology. Circ Cardiovasc Interv 2014; 7: 425-8. 